\title{
Local incompatibility of the microlocal spectrum condition with the KMS property along spacelike directions in quantum field theory on curved spacetime
}

\author{
Nicola Pinamonti ${ }^{1,2, a}$, Ko Sanders ${ }^{3, b}$, Rainer $\operatorname{Verch}^{4, c}$ \\ ${ }^{1}$ Dipartimento di Matematica, Università di Genova - Via Dodecaneso, 35, I-16146 Genova, Italy. \\ ${ }^{2}$ Istituto Nazionale di Fisica Nucleare - Sezione di Genova, Via Dodecaneso, 33 I-16146 Genova, Italy. \\ ${ }^{3}$ School of Mathematical Sciences and Centre for Astrophysics and Relativity, Dublin City University, \\ Glasnevin, Dublin 9, Ireland. \\ ${ }^{4}$ Institute for Theoretical Physics, University of Leipzig, D-04009 Leipzig, Germany. \\ E-mail: ${ }^{a}$ pinamont@dima.unige.it, ${ }^{b}$ jacobus.sanders@dcu.ie, ${ }^{c}$ rainer.verch@uni-leipzig.de
}

Version of June 10, 2019

\begin{abstract}
States of a generic quantum field theory on a curved spacetime are considered which satisfy the KMS condition with respect to an evolution associated with a complete (Killing) vector field. It is shown that at any point where the vector field is spacelike, such states cannot satisfy a certain microlocal condition which is weaker than the microlocal spectrum condition in the case of asymptotically free fields.
\end{abstract}

\section{Introduction}

In the standard framework of quantum statistical mechanics [3] the observables of a quantum system are described by the self-adjoint elements of a non-commutative $C^{*}$-algebra $\mathcal{A}$ with a unit element 1. A time-evolution is described by a one-parameter group of $*$-automorphisms $\left\{\alpha_{t}\right\}_{t \in \mathbb{R}}$ acting on $\mathcal{A}$ that is (weakly) continuous in $t$. Together, $\mathcal{A}$ and $\left\{\alpha_{t}\right\}_{t \in \mathbb{R}}$ form a $C^{*}$ dynamical system. In this framework, a state $\omega$ on $\mathcal{A}$ is described by a linear functional which is normalized, $\omega(\mathbf{1})=1$, and positive, $\omega\left(A^{*} A\right) \geq 0(A \in \mathcal{A})$. The evaluation of $\omega$ on an observable $A \in \mathcal{A}$, denoted $\omega(A) \equiv\langle A\rangle_{\omega}$, is interpreted as the expectation value.

The class of all states on a $C^{*}$-dynamical system is very large and it is known from examples that there are states that correspond to unphysical scenarios like infinite energy densities or infinite pressures. For this reason it is essential to select a class of simple states that do correspond to physically realistic configuations of a system. A particularly important class is furnished by states which describe systems in equilibrium with a thermal bath at a fixed temperature. Such states are characterized by a very small number of parameters, like their temperature and chemical potential. Thus, for such states, the expectation values of physically relevant observables are functions of these parameters, and furthermore, relations between different expectation values are described by equations of thermodynamical (thermostatic) nature. Prime examples of 
equilibrium states are the well-known Gibbs states [11, which are used to model the canonical ensemble of quantum particles at fixed temperature in a box. A generalization of Gibbs states is the class of states that satisfy the Kubo-Martin-Schwinger (KMS) condition [12 with respect to the system's time-evolution $\left\{\alpha_{t}\right\}_{t \in \mathbb{R}}$ (see Definition 2.1). States which satisfy the KMS condition are called KMS states, and they can also be defined for more general settings, where e.g. $\mathcal{A}$ may be a more general *-algebra than a $C^{*}$-algebra.

The KMS condition is so versatile that it can be used to characterize equilibrium states for non-relativistic or relativistic quantum field theories both on flat and curved spacetimes. In this letter we are interested in analyzing KMS states for relativistic quantum field theories on a (possibly curved) spacetime $M$ with spacetime metric $g$. In this case, the simplest concept of time-evolution, generalizing the time-translations on Minkowski spacetime, is tied to spacetimes with a smooth Killing vector field $\chi$, which generates a one-parametric group of isometries for the spacetime metric. In order to interpret the group of isometries as time-translations, it is natural to assume that $\chi$ is everywhere timelike, so the spacetime is stationary. However, it is interesting to notice that it is not uncommon for spacetimes to admit isometry groups generated by Killing vector fields which are timelike in some regions of spacetime and spacelike in other regions. The most prominent example is furnished by the rotating Kerr black hole [29], whose outer horizon is the bifurcate Killing horizon of a Killing vector field that is timelike just outside of the outer horizon, but which becomes spacelike far away from and inside of the black hole region. This prompts the obvious question if global KMS states with respect to the corresponding one-parameter evolution exist in such cases.

As will be outlined in Section 3 a well-defined quasifree KMS state with respect to spacelike translations can be constructed for free Fermi field:1 on flat spacetime, however, we will see that the obtained state is not admissible in a sense to be discussed below (see Section 2 for the full definition) 2 In particular, the state will not be of Hadamard type. For free bosonic fields on Minkowski spacetime the very same construction does not lead to the definition of a well posed two-point function of a state because of infrared divergences. For general quantum field theories on Minkowski spacetime, satisfying locality and translation invariance, it follows from the work of Trebels [27] that a vacuum state cannot be a KMS state for the flow of a Killing field $\chi$ in regions where $\chi$ is spacelike. (This can happen when $\chi$ is timelike, e.g. in the Unruh effect, cf. [30.)

In the setting of curved spacetimes, for the particular case of the Kerr spacetime and a free quantized scalar field, satisfying the covariant Klein-Gordon equation, Kay and Wald invoked a superradiance property to argue that Killing field invariant Hadamard states do not exist [17. For a class of states which satisfy the KMS condition they note that the superradiance

\footnotetext{
${ }^{1}$ Throughout this paper we will call a theory free when the dynamical fields satisfy a linear field equation.

${ }^{2} \mathrm{~A}$ very general statement on the inconsistency of the KMS condition for spacelike translations is made in [2, but there appears to be an error in the proof of Lemma 3.6 of that reference concerning entire holomorphic functions of exponential type. The statement in 2 would rule out the aforementioned Fermi field KMS states for spacelike translations on the even part of the Fermi field algebra; however the result in Sec. 3 shows they exist.
} 
assumption may be dropped. Indeed, implicitly they have shown that for a quantized KleinGordon field on any globally hyperbolic spacetime with a Killing field $\chi$ that becomes spacelike there can be no quasifree KMS states satisfying additional regularity conditions (see the corollary to Lemma 6.2 and the first two paragraphs of Section 6.4 in loc.cit.) 3

In this regard, it is worth noting that the microlocal spectrum condition, a generalization of the Hadamard condition, is by now recognized to be an unavoidable requirement for states of quantum fields to be viewed as physically realistic [5, 8, 7, 30. Moreover, KMS states for linear quantum fields on a stationary spacetime, where the corresponding Killing vector field is everywhere timelike, have been shown to fulfill the microlocal spectrum condition [20]. The existence of KMS states for quantized linear fields on stationary spacetimes has also been established, see 24] and references cited therein.

In this note we show that KMS states cannot be admissible in the neighborhood of points where the vector field $\chi$ is spacelike. No particular form of field equation needs to be assumed for the quantum field, i.e. the argument is model-independent. The term "admissible", defined in Sec. 2, is closely related to the microlocal spectrum condition and for free fields it is equivalent to it. Neither the regularity conditions on the KMS state used in [17], nor the global hyperbolicity of $M$ nor the Killing property of $\chi$ are required in our proof.

To motivate the admissibility condition that we shall impose on states of a quantum field on a curved spacetime in Sec. 2, let us first briefly recall here the microlocal spectrum condition in its simplest form, i.e. for a scalar quantum field $\phi$. The spacetime $M$ is assumed to be time-oriented and globally hyperbolic. The $*$-algebra $\mathcal{A}$ of observables is generated by the set $\{\phi(f) \mid f \in \mathcal{D}(M)\}$ formed by quantum field operators smeared with smooth test functions. A state $\omega$ on $\mathcal{A}$ is determined by the $n$-point functions $w_{n}^{\omega}\left(f_{1}, \ldots, f_{n}\right)=\omega\left(\phi\left(f_{1}\right) \cdots \phi\left(f_{n}\right)\right)$ $\left(f_{j} \in \mathcal{D}(M)\right)$ which, by definition, are (or rather, extend to) distributions in $\mathcal{D}\left(M^{n}\right)$. The microlocal spectrum condition for a state $\omega$ is a condition of the form $\mathrm{WF}\left(w_{n}^{\omega}\right) \subset \Gamma_{n}$ on the wave front sets of the distributions $w_{n}^{\omega}$. We refer to [15] for the concept of wave front set for distributions on a manifold, and to [5] for the definition of the sets $\Gamma_{n}$ in the microlocal spectrum condition. Informally, this condition imposes an upper bound on the allowed singularities of the $n$-point functions.

The microlocal spectrum condition is motivated by the work of Radzikowski [19], who showed that for a free scalar field the Hadamard condition [17, 30] on the two-point function $w_{2}^{\omega}$ is equivalent to the microlocal condition 4

$$
\begin{aligned}
\mathrm{WF}\left(w_{2}^{\omega}\right)= & \left\{\left(x,-k ; x^{\prime}, k^{\prime}\right) \in T^{*} M \times T^{*} M \backslash\{\mathbf{0}\} \mid\left(x^{\prime}, k^{\prime}\right) \in \mathcal{N}^{-},\right. \\
& \left.(x, k) \sim\left(x^{\prime}, k^{\prime}\right)\right\}
\end{aligned}
$$

\footnotetext{
${ }^{3}$ We are grateful to an anonymous referee for pointing out this implicit result.

${ }^{4}$ Here, $\mathbf{0}$ denotes the zero section in $T^{*} M \times T^{*} M$. Writing $(x, k)$ for an element in $T^{*} M$ means that $k \in T_{x}^{*} M$, i.e. $x$ denotes the point in the base manifold $M$ at which the cotangent vector $k$ is "affixed".
} 
where $\mathcal{N}^{-}$is the set of past-directed lightlike co-vectors of $M$, i.e. $g^{-1}\left(k^{\prime}\right)$ is a lightlike vector and $k(v)<0$ for any future pointing tangent vector $v$ of $M$ at $x$ Furthermore, $(x, k) \sim\left(x^{\prime}, k^{\prime}\right)$ means that $x$ and $x^{\prime}$ are joined by a null geodesic $\gamma$ and $g^{-1}(k)$ and $g^{-1}\left(k^{\prime}\right)$ are tangent to $\gamma$ and coincide up to parallel transport along $\gamma$. Analogous statements have been proved for other types of quantized fields that are subject to linear hyperbolic field equations [21. Moreover, for such fields, (1) is equivalent to the 2-point part of the microlocal spectrum condition, $\operatorname{WF}\left(w_{2}^{\omega}\right) \subset \Gamma_{2}$ [26], and for any fields satisfying canonical commutation or anti-commutation relations, it is equivalent to the full microlocal spectrum condition [22]. The microlocal spectrum condition has also been generalized to encompass the extended algebra of Wick polynomials of free fields on flat and curved spacetimes [5] and the use of microlocal techniques has opened up the possibility of a local covariant perturbative construction of interacting field theories on curved spacetimes [4, 13, 14. In [8] it has been shown that the microlocal spectrum condition is not only sufficient, but also necessary for a state to be well-defined on the extended algebra of Wick polynomials. In the light of these results, demanding that states satisfy the microlocal spectrum condition can surely be viewed as non-negotiable in local covariant quantum field theory.

Worth noting is the equality in (1): the specification of $\mathrm{WF}\left(w_{2}^{\omega}\right)$ is a restriction both from "above" and "below", which is stronger than the condition $\operatorname{WF}\left(w_{2}^{\omega}\right) \subset \Gamma_{2}$. The admissibility condition that we shall impose in Sec. 2 is in fact a lower bound on $\operatorname{WF}\left(w_{2}^{\omega}\right)$, namely that it contains all lightlike co-vector pairs $(x,-k ; x, k)$ with $(x, k) \in \mathcal{N}^{-}$. This condition follows from (11), so it holds for free fields. More generally it is a local and covariant remnant of the Lorentz covariance of the spectrum condition familiar from quantum field theory in Minkowski spacetime.

In the following section we will delineate the precise assumptions and present the proof.

\section{Result}

We consider a spacetime $(M, g)$, where $M$ is a four dimensional, oriented and time-oriented, smooth manifold and $g$ is a Lorentzian metric of signature $(-,+,+,+)$. An additional assumption could be that $(M, g)$ is globally hyperbolic, but our proof below does not require it. Furthermore, we consider a non-commutative $*$-algebra $\mathcal{A}$ generated by a unit element $\mathbf{1}$ and "smeared quantum field operators" $\phi(F)$ where $F$ stands for any smooth, compactly supported section of a given complex vector bundle $E$ over $M$ of some finite dimension $N 6$ We denote by $\Gamma$ the action of a fibrewise anti-linear involution on $E$ and we also assume that it induces a continuous map $\Gamma: \mathcal{D}(E) \rightarrow \mathcal{D}(E)$, where $\mathcal{D}(E)$ denotes the space of smooth, compactly supported sections of $E$, equipped in the usual manner with the $\mathcal{D}$-topology. For simplicity we also impose $\phi(F)^{*}=\phi(\Gamma F)$.

We also assume that $E$ admits a complete smooth vector field $V$ which projects down to a

\footnotetext{
${ }^{5}$ Using index notation, $g^{-1}(k)$ reads $g^{\mu \nu} k_{\nu}$, and $k(v)$ reads $k_{\mu} v^{\mu}$.

${ }^{6}$ The $\phi(F)$ need not be represented as operators on some Hilbert space, but may well be elements of an "abstract" algebra.
} 
smooth, complete vector field $\chi$ on $(M, g)$. These vector fields generate one-parameter groups of diffeomorphisms, $\left\{\eta_{t}\right\}_{t \in \mathbb{R}}$ on $E$ and $\left\{\tau_{t}\right\}_{t \in \mathbb{R}}$ on $M$. Furthermore, we assume that $\Gamma$ commutes with $\eta_{t}$ and that the diffeomorphism groups induce a one-parametric evolution group of *automorphisms $\left\{\alpha_{t}\right\}_{t \in \mathbb{R}}$ on $\mathcal{A}$ by

$$
\alpha_{t}(\phi(F)):=\phi\left(\Psi_{t}(F)\right), \quad \Psi_{t}(F):=\eta_{-t} \circ F \circ \tau_{t} .
$$

Then $\mathcal{A}$ together with $\left\{\alpha_{t}\right\}_{t \in \mathbb{R}}$ is a $*$-algebraic dynamical system. For our discussion it is not necessary to be more specific than this. In particular, we don't assume any field equations to be fulfilled by the $\phi(F)$. The hypotheses we are imposing are completely general and are satisfied by various free or interacting fermionic or bosonic fields.

Consider a state $\omega$ on $\mathcal{A}$, i.e. a linear functional on $\mathcal{A} \rightarrow \mathbb{C}$ which is positive $\left(\omega\left(A^{*} A\right) \geq 0\right.$ for all $A \in \mathcal{A})$ and normalized $(\omega(\mathbf{1})=1)$. Let us write the associated two-point function as

$$
w_{2}^{\omega}\left(F, F^{\prime}\right):=\omega\left(\phi(F) \phi\left(F^{\prime}\right)\right) .
$$

We assume that the map

$$
\left(F, F^{\prime}\right) \mapsto w_{2}^{\omega}\left(F, F^{\prime}\right)
$$

gives rise to a distribution over compactly supported smooth section: $7 \mathcal{7}(E) \otimes \mathcal{D}(E)$ of the outer product bundle $E \otimes E$ over $M \times M$ (see e.g. [20] Sec.3.3 for a definition).

Below, it will turn out useful to consider a Hilbert space-valued distribution associated with the two-point function $w_{2}^{\omega}$ which is obtained by the GNS construction (see e.g. [9] for a description of the GNS representation of a unital $*$-algebra associated with a state). For the sake of self-containedness, we describe how that Hilbert space-valued distribution is obtained. The positivity of the state implies that

$$
w_{2}^{\omega}(\Gamma F, F) \geq 0
$$

so that $w_{2}^{\omega}$ defines a semi-definite sesquilinear form on $\mathcal{D}(E)$ by $\left(F, F^{\prime}\right) \mapsto w_{2}^{\omega}\left(\Gamma F, F^{\prime}\right)$. The sections $F \in \mathcal{D}(E)$ with $w_{2}^{\omega}(\Gamma F, F)=0$ form a linear space $K$, by the Cauchy-Schwarz inequality. We denote the equivalence classes in $\mathcal{D}(E) / K$ by $[F]$ and we complete this quotient space to a Hilbert space $\mathcal{H}$ using the inner product

$$
\left\langle[F],\left[F^{\prime}\right]\right\rangle:=w_{2}^{\omega}\left(\Gamma F, F^{\prime}\right) .
$$

It will be convenient to consider the $\mathcal{H}$-valued map $\mathcal{D}(E) \rightarrow \mathcal{H}$ defined by $F \mapsto[F]$, which we will write as

$$
F \mapsto \phi(F) \Omega .
$$

\footnotetext{
${ }^{7}$ Here we really mean $\mathcal{D}(E \otimes E)$, which is the completion of $\mathcal{D}(E) \otimes \mathcal{D}(E)$ in the (unique) locally convex topology derived from the (nuclear) test-function topology on $\mathcal{D}(E)$.
} 
For good reason, this is reminiscent of the GNS representation; $\phi(F)$ is the representer of $\phi(F)$ in the GNS representation and $\Omega$ is the GNS vector. We note that

$$
\left\langle\phi(F) \Omega, \phi\left(F^{\prime}\right) \Omega\right\rangle=w_{2}^{\omega}\left(\Gamma F, F^{\prime}\right)
$$

and that the map $F \mapsto \phi(F) \Omega$ is an $\mathcal{H}$-valued distribution, because $w_{2}^{\omega}$ is a distribution; see [26] for details.

Definition 2.1. A state $\omega$ on $\mathcal{A}$ satisfies the $K M S$ condition with respect to $\left\{\alpha_{t}\right\}_{t \in \mathbb{R}}$ at inverse temperature $\beta$ if, for every $A, B \in \mathcal{A}$ the function

$$
t \mapsto \omega\left(A \alpha_{t}(B)\right) \quad(t \in \mathbb{R})
$$

has a continuous and bounded extension to the closed strip

$$
S_{\beta}=\{\mathrm{t}=t+i s \in \mathbb{C} \mid 0 \leq s \leq \beta, t \in \mathbb{R}\}
$$

which is analytic in the open interior of $S_{\beta}$ and satisfies

$$
\omega\left(A \alpha_{t+i \beta}(B)\right)=\omega\left(\alpha_{t}(B) A\right) \quad(t \in \mathbb{R}) .
$$

The state $\omega$ is then called a KMS state at inverse temperature $\beta$ with respect to the oneparametric evolution group $\left\{\alpha_{t}\right\}_{t \in \mathbb{R}}$. (Cf. e.g. [18] and references cited there; the KMS condition carries over from the $C^{*}$-algebra setting in an obvious way to states and continuous automorphism groups on more general unital *-algebras.)

One can show that KMS states are necessarily invariant under all the $\alpha_{t}$. This invariance is typically incompatible with the dynamics of the field, unless the evolution $\tau_{t}$ is given by isometries of the spacetime, i.e. $\chi$ is a Killing field. For our result, however, this Killing property will not be required.

If $\omega$ is a KMS state at inverse temperature $\beta$, then the 2-point function satisfies properties analogous to those listed in Def. 2.1, in particular $w_{2}^{\omega}$ is invariant under the action of $\eta_{t}$ and $\tau_{t}$ in the sense that

$$
w_{2}^{\omega}\left(\Psi_{t}(F), \Psi_{t}\left(F^{\prime}\right)\right)=w_{2}^{\omega}\left(F, F^{\prime}\right)
$$

and the KMS condition implies that for any $F, F^{\prime} \in \mathcal{D}(E)$ the map

$$
t \mapsto w_{2}^{\omega}\left(F, \Psi_{t}\left(F^{\prime}\right)\right)
$$

has a bounded and continuous extension to the strip $S_{\beta}$, analytic in the interior, such that

$$
w_{2}^{\omega}\left(F, \Psi_{t+i \beta}\left(F^{\prime}\right)\right)=w_{2}^{\omega}\left(\Psi_{t}\left(F^{\prime}\right), F\right) \quad(t \in \mathbb{R}) .
$$

Definition 2.2. We say that a state $\omega$ on $\mathcal{A}$ is admissible at $x \in M$ if

$$
\mathrm{WF}(\phi(.) \Omega) \cap T_{x}^{*} M \supset \mathcal{N}_{x}^{-},
$$

where $\mathcal{N}_{x}^{-}=\mathcal{N}^{-} \cap T_{x}^{*} M$ and $\mathcal{N}^{-} \subset T^{*} M$ is the set of past-directed lightlike co-vectors. We call $\omega$ admissible if it is admissible at all $x \in M$, or equivalently when

$$
\mathrm{WF}\left(w_{2}^{\omega}\right) \supset\left\{(x,-k ; x, k) \mid(x, k) \in \mathcal{N}^{-}\right\} .
$$


We note that $\operatorname{WF}(\phi(.) \Omega)$ is the wave front set of the Hilbert space-valued distribution $F \mapsto$ $\phi(F) \Omega$, cf. [26]. The equivalence in the definition follows from Prop. 6.1 of [26]. As discussed in the Introduction, for the quantized Klein-Gordon field the microlocal spectrum condition at the level of 2-point functions implies this admissibility condition (cf. eqn. (10) and this holds also for other types of quantized fields that are subject to a linear hyperbolic equation, like the Dirac field and the vector potential [21].

Our admissibility condition can even be expected for many reasonable interacting theories. Indeed, let us suppose that the theory is asymptotically free in the sense that at every point $x \in M$ the state $\omega$ has a short-distance scaling limit $\omega_{x}$ at $x[9]$, such that the scaling limit state $\omega_{x}$ is the vacuum state of a free quantized field on $T_{x} M \simeq$ Minkowski spacetime. Combining Prop. A.2 of [23] (see also Prop. 2.8 of [21], cf. also [28]) for scaling limits of distributions with the results of [26] we find in this case

$$
\mathrm{WF}(\phi(.) \Omega) \supset\left\{(x, k) \mid(0, k) \in \mathrm{WF}\left(\phi_{0}(.) \Omega_{x}\right)\right\} \supset \mathcal{N}^{-} \cap T_{x}^{*} M .
$$

where $\phi_{0}$ refers to the scaling limit quantum field. Taking the union over all $x \in M$ we conclude that $\omega$ is admissible.

Theorem. Consider a KMS state $\omega$ at inverse temperature $\beta$ with respect to the evolution induced by the complete smooth vector field $V$ on $E$ with projection $\chi$ on $M$. If $\chi$ is spacelike at a point $x \in M$, then $\omega$ is not admissible at the point $x$.

For quantum fields satisfying a linear hyperbolic equation, the proof of this statement is actually contained in the proof of Thm. 5.1 of [20] (the assumption made in this reference that the Killing vector field is timelike does not enter in the proof). However, here we give a different proof.

Proof. Since every $\beta$-KMS state is invariant under the evolution $\Psi_{t}$, there is a strongly continuous unitary group $U(t)=e^{i t H}$ on $\mathcal{H}$, generated by a self-adjoint operator $H$, such that

$$
e^{i t H} \phi(F) \Omega=\phi\left(\Psi_{t}(F)\right) \Omega
$$

for all $F \in \mathcal{D}(E)$. Moreover, due to the $\beta$-KMS condition, the map

$$
t \mapsto e^{i t H} \phi(F) \Omega
$$

is bounded and continuous on the strip $S_{\beta / 2}$ and holomorphic on the interior ([18] Prop. 8.14.2).

Now consider a coordinate system $(\xi, \mathcal{O})$ which contains $x$ and is adapted to $\tau_{t}$. This means that for any point $y \in \mathcal{O}$ with $\xi(y)=\left(y^{0}, y^{1}, y^{2}, y^{3}\right)$ we have

$$
\xi\left(\tau_{t} y\right)=\left(y^{0}, y^{1}+t, y^{2}, y^{3}\right), \quad \text { for all } t \in \mathbb{R} \text { such that } \tau_{t} y \in \mathcal{O} .
$$

Shrinking $\mathcal{O}$ if necessary we may also introduce a frame $\left\{e_{1}, \ldots, e_{N}\right\}$ for $E$ on $\mathcal{O}$ such that $\Psi_{t}\left(e_{j}\right)=e_{j}$ for each $j=1, \ldots, N$ at all points where both sides are defined. (We may first 
choose such a frame on the hypersurface $y^{1}=0$ and then use the evolution $\Psi_{t}$ to extend it.) For each $j=1, \ldots, N$ we consider the $\mathcal{H}$-valued distribution $G_{j}$ on $(0, \beta / 2) \times \mathcal{O}$ defined by

$$
G_{j}(\eta, f)=e^{-\eta H} \phi\left(f e_{j}\right) \Omega,
$$

where $f$ is in $\mathcal{D}(\mathcal{O}) . G_{j}$ is seen to satisfy $\left(\partial_{y}+i \partial_{\eta}\right) G_{j}=0$, so it is holomorphic in $y+i \eta$, and its boundary value as $\eta \rightarrow 0$ is the distribution $f \mapsto \phi\left(f e_{j}\right) \Omega$. Although $G_{j}$ is not a smooth function, we can still conclude as in Thm. 2.8 of [26] that

$$
\mathrm{WF}\left(\phi\left(. e_{j}\right) \Omega\right) \cap T_{x}^{*} M \subset\left\{(x, k) \in T^{*} M \mid k(\chi) \geq 0\right\} ;
$$

in fact, the proof of Thm. 2.8 of [26] can be seen by inspection to generalize to the case that the $G_{j}($.$) are distributions analytic in y+i \eta$, on observing that $\left|G_{j}(\eta, y, f)\right| \leq C\|f\|_{m}$ for a Sobolev norm of sufficiently high degree $m$, for all $f\left(y^{0}, y^{2}, y^{3}\right)$ supported in a fixed compact set and a constant $C>0$, uniformly in $(\eta, y)$. (See also Thm. 8.1.6 of [15].)

Combining the components $\phi\left(. e_{j}\right) \Omega$ of the distribution $\phi(.) \Omega$ we find (cf. [21])

$$
\mathrm{WF}(\phi(.) \Omega) \cap T_{x}^{*} M \subset \bigcup_{j=1}^{N} \mathrm{WF}\left(\phi\left(. e_{j}\right) \Omega\right) \cap T_{x}^{*} M \subset\left\{(x, k) \in T^{*} M \mid k(\chi) \geq 0\right\} .
$$

However, when $\chi$ is spacelike at $x$, there is a dual null vector $k$ at $x$ such that $k(\chi)<0$, so we cannot have $\mathcal{N}^{-} \subset \mathrm{WF}(\phi(.) \Omega)$, i.e. $\omega$ cannot be admissible at $x$.

\section{The case of Fermi fields on flat spacetime}

In this section we shall discuss the existence of KMS states for Fermi fields with respect to spacelike translations in a four dimensional Minkowski spacetime. The state we shall obtain will however be inadmissible in the sense of Section 2 .

In order to construct the desired KMS state for free Fermi fields we need just consider the two-point function, because we choose the state to be quasi-free. This two-point function can be explicitly constructed as follows. Consider a free Dirac field $\psi$ on Minkowski spacetime 8 The two-point function of a KMS state $\omega$ with respect to spacelike translations along the direction determined by the normalised spatial vector $e_{j}$ and implemented by the one parameter group of $*$-automorphisms $\alpha_{s}(\psi(x))=\psi\left(x-s e_{j}\right)$ can be constructed out of the anticommutator function $S(x, y)=\omega\left(\psi(x) \psi^{\dagger}(y)\right)+\omega\left(\psi^{\dagger}(y) \psi(x)\right)$ which defines the CAR relations. Since $S$ is a translation-invariant Schwartz distribution we can consider its Fourier transform $\hat{S}$ and multipliying it with the corresponding Fermi factors we obtain

$$
\hat{w}_{2}^{\omega+}(k)=\frac{\hat{S}(k)}{e^{-\beta k_{j}}+1}, \quad \hat{w}_{2}^{\omega-}(k)=\frac{\hat{S}(k)}{e^{+\beta k_{j}}+1},
$$

\footnotetext{
${ }^{8}$ We refer to 23 for details about the quantization of Dirac fields and for the form of the distribution $S$ implementing the CAR.
} 
where $k_{j}=\left\langle k, e_{j}\right\rangle$. The functions $e^{ \pm i z k_{j}}\left(e^{\mp \beta k_{j}}+1\right)^{-1}$ are smooth, bounded functions on $\mathbb{R}^{4}$ for every $\operatorname{Im}(z) \in[0, \beta]$, furthermore, they are positive for $z=0$, hence also $\hat{w}_{2}^{\omega \pm}(k)$ are Schwartz distributions. Furthermore, their inverse Fourier transforms define the two-point functions of a quasi-free state $\omega\left(\psi(x) \psi^{\dagger}(y)\right)=w_{2}^{\omega+}(x-y)$ and $\omega\left(\psi^{\dagger}(y) \psi(x)\right)=w_{2}^{\omega-}(x-y)$. This state is a KMS state with respect translations along the direction $e_{j}$ at inverse temperature $\beta$, however it cannot be a Hadamard state as proved in Section 2 .

For quantized bosonic fields on Minkowski spacetime fulfilling a linear hyperbolic field equation, a similar procedure cannot be applied. Actually, if one tries to construct the relevant two-point functions of extremal KMS states multiplying the commutator function with the appropriate Bose factor in the Fourier domain along the lines of [6], some divergences are encountered. These divergences are due to the fact that the Bose factor with respect to spacelike translations is not locally integrable in momentum space.

\section{Summary and Outlook}

We have shown that for quantum fields on curved spacetimes obeying minimal assumptions, a microlocal admissibility condition for the 2-point function of a state is locally incompatible with the KMS condition with respect to a spacelike evolution group. For free or asymptotically free fields this implies an incompatibility with the microlocal spectrum condition, which is stronger.

As indicated, there are examples of spacetimes that are considered to be of physical relevance, such as the rotating Kerr black hole spacetimes, possessing Killing vector fields which are timelike in some (accessible) region of spacetime and spacelike in other (accessible) regions. Therefore, quantum field states that fulfill the admissibility condition can only satisfy the KMS condition in the regions of spacetime where the Killing vector field of the evolution group is not spacelike. There are examples for such behaviour already in Minkowski spacetime: By the BisognanoWichmann Theorem [1], the vacuum state of any Wightman-type quantum field theory restricts to a KMS state at inverse temperature $2 \pi$ with respect to the action of a one parametric group of Lorentz boosts on the algebra of field operators localized in the Rindler wedge region $W$ of spacetime containing all boost trajectories such that the associated Killing vector field is timelike and future-directed. On the algebra of the causal complement $-W$ of that region, the vacuum state restricts to a KMS-state at inverse temperature $-2 \pi$ with respect to the same one-parametric group of Lorentz boosts. In the (open) complement of $W \cup-W$ in Minkowski spacetime, the trajectories of the one-parametric group of Lorentz boosts are spacelike, and the vacuum state has no KMS-like properties in restriction to operators localized in that complement region. A completely analogous situation occurs for quantum fields on the Schwarzschild-Kruskal spacetime [16, 17, 25]. In the case of the Kerr rotating black hole spacetime, where the Killing vector field in the exterior of the black hole region changes between timelike and spacelike, it is still possible that there exist quantum field states that fulfill the admissibility and microlocal spectrum condition and have KMS-like properties in the region where the Killing vector field is timelike (a local generalization of the KMS condition has been given, see e.g. [10] and references cited there). Our method of proof does a priori not rule out the possibility of quantum field 
states that fulfill the admissibility and microlocal spectrum condition and the KMS condition with respect to an evolution group with a lightlike Killing vector field, and it would be interesting to investigate that possibility further.

\section{Acknowledgements}

N.P. thanks the Institute for Theoretical Physics of the University of Leipzig for the kind hospitality during the preparation of this work and the DAAD for supporting this visit with the program "Research Stays for Academics 2017". K.S. thanks Christian Gérard for bringing his notes on this result to the attention of N.P. and R.V.

\section{References}

[1] Bisognano, J.J., Wichmann, E.H.: On the duality condition for quantum fields. J. Math. Phys. 17, 303-321 (1976)

[2] Borchers, H.J.: Theory of Local Observables and KMS Condition. Preprint, Institut für Theoretische Physik, University of Göttingen, 1994 https://pdfs. semanticscholar.org/de96/92cd57c951816af536d90cdb38c718e9ddf2.pdf

[3] Bratteli, O., Robinson, D.W.: Operator Algebras and Quantum Statistical Mechanics, Vols. 1 and 2. Springer Verlag Berlin Heidelberg, 1987 and 1997

[4] Brunetti, R., Fredenhagen, K.: Microlocal analysis and interacting quantum field theories: Renormalization on physical backgrounds. Commun. Math. Phys. 208, 623 (2000)

[5] Brunetti, R., Fredenhagen, K., Köhler, M.: The microlocal spectrum condition and Wick polynomials of free fields on curved spacetimes. Commun. Math. Phys. 180, 633 (1996)

[6] Buchholz, D., Verch, R.: Macroscopic aspects of the Unruh effect. Class. Quant. Grav. 32, 245004 (2015)

[7] Fewster, C.J., Verch, R.: Stability of quantum systems at three scales: Passivity, quantum weak energy inequalities and the microlocal spectrum condition. Commun. Math. Phys. 240, 329-375 (2003)

[8] Fewster, C.J., Verch, R.: The necessity of the Hadamard condition. Class. Quantum Grav. 30, 235027 (2013)

[9] Fredenhagen, K., Haag, R.: Generally covariant quantum field theory and scaling limits. Commun. Math. Phys. 108, 91 (1987)

[10] Gransee, M., Pinamonti, N., Verch, R.: KMS-like properties of local equilibrium states in quantum field theory. J. Geom. Phys. 117, 15-35 (2017) 
[11] Haag, R.: Local quantum physics. Second edition, Springer-Verlag Berlin, 1992 ISBN: 3-540-61451-6.

[12] Haag, R., Hugenholtz, N., Winnink, M.: On the equilibrium state in quantum statistical mechanics. Commun. Math. Phys. 5, 215-236 (1967)

[13] Hollands, S., Wald, R.M.: Local Wick polynomials and time ordered products of quantum fields in curved spacetime. Commun. Math. Phys. 223, 289 (2001)

[14] Hollands, S., Wald, R.M.: Existence of local covariant time ordered products of quantum fields in curved spacetime. Commun. Math. Phys. 231, 309 (2002)

[15] Hörmander, L.: The Analysis of Linear Partial Differential Operators I. Second edition, Springer-Verlag Berlin, 1989 ISBN: 3-540-00662-1.

[16] Kay, B.S.: The double wedge algebra for quantum fields on Schwarzschild and Minkowski space-times. Commun. Math. Phys. 10057 (1985)

[17] Kay, B.S., Wald, R.M.: Theorems on the uniqueness and thermal properties of stationary, nonsingular, quasifree states on spacetimes with a bifurcate Killing horizon. Physics Reports, 207, 49-136 (1991)

[18] Pedersen, G.K.: $C^{*}$-Algebras and their Automorphism Groups. Lecture Notes in Mathematics, Academic Press (1979)

[19] Radzikowski, M.J.: Micro-local approach to the Hadamard condition in quantum field theory on curved space-time. Commun. Math. Phys. 179, 529 (1996)

[20] Sahlmann, H., Verch, R.: Passivity and Microlocal Spectrum Condition. Commun. Math. Phys. 214, 705-731 (2000)

[21] Sahlmann, H., Verch, R.: Microlocal spectrum condition and Hadamard form for vectorvalued quantum fields in curved spacetime. Rev. Math. Phys. 13, 1203-1246 (2001)

[22] Sanders, K.: Equivalence of the (generalised) Hadamard and Microlocal Spectrum Condition for (generalised) free fields in curved spacetime. Commun. Math. Phys. 295, 485-501 (2010)

[23] Sanders, K.: The locally covariant Dirac field. Rev. Math. Phys. 22, 381-430 (2010)

[24] Sanders, K.: Thermal equilibrium states of a linear scalar quantum field in stationary spacetimes. Internat. J. Modern Phys. A 28, 1330010 (2013)

[25] Sanders, K.: On the construction of Hartle-Hawking-Israel states across a static bifurcate Killing horizon. Lett. Math. Phys. 105, 575-640 (2015) 
[26] Strohmaier, A., Verch, R., Wollenberg, M.: Microlocal analysis of quantum fields on curved space-times: Analytic wave front sets and Reeh-Schlieder theorems. J. Math. Phys. 43, 5514-5530 (2002)

[27] Trebels, S.: Über die geometrische Wirkung modularer Automorphismen: Analyse in Algebraischer Quantenfeldtheorie. PhD Thesis, Dept. of Physics, University of Göttingen (1997)

[28] Verch, R.: Wavefront sets in algebraic quantum field theory. Commun. Math. Phys. 205, 337-367 (1999)

[29] Wald, R.M.: General Relativity. University of Chicago Press (1984)

[30] Wald, R.M.: Quantum Field Theory in Curved Space-Time and Black Hole Thermodynamics. University of Chicago Press (1995) 